\title{
Development and Evaluation of a CT Pulmonary Angiography Protocol Dedicated to Pregnant and Postpartum Women
}

\author{
Alfredo Cantarinha, $\mathrm{RT}^{\mathrm{ab} *}$, Jean-Philippe Dillenseger, RT, $\mathrm{PhD}^{\mathrm{bcd}}$ and \\ Marie France Bellin, MD, $\mathrm{PhD}^{\mathrm{a}}$ \\ ${ }^{a}$ Póle d'imagerie médicale, $\mathrm{CHU}$ du Kremlin Bicêtre, Paris, France \\ ${ }^{\mathrm{b}}$ Collège scientifique de l'Association française du personnel paramédical d'electroradiologie médicale (AFPPE), Paris, France \\ ' Section Imagerie Médicale et Radiologie Thérapeutique, Académie de Strasbourg, Strasbourg, France \\ ${ }^{\mathrm{d}}$ ICube - UMR 7357, CNRS, Université de Strasbourg, Strasbourg, France
}

\begin{abstract}
Introduction: This study presents and evaluates a CT pulmonary angiography protocol dedicated to pregnant women. The specific feature of this protocol is to place the region of interest (ROI) (bolus detection) in the superior vena cava. The objective is to evaluate the performances of this method.
\end{abstract}

Materials and methods: The protocol uses a iodine-based contrast agent at $300 \mathrm{mgI} / \mathrm{mL}$ and an injection rate of 5 to $6 \mathrm{~mL} / \mathrm{sec}$ for an injection volume of $50 \mathrm{~mL}$ of iodine contrast agent followed by $40 \mathrm{~mL}$ of $\mathrm{NaCl}$. The ROI is positioned on the superior vena cava, with a 100 Hounsfield units (HU) threshold, and the acquisition is performed at $100 \mathrm{kVp}$. This protocol was evaluated retrospectively on a large population $(\mathrm{n}=105$ : group 1$)$ and compared with a control group that did not benefit from this protocol $(n=55$ : group 2$)$. Both groups were studied on the same device in the same center. Each examination was evaluated and classified into 3 groups: optimal, suboptimal, and noncontributory. Dose length products (DLP) values were also recorded. Statistical tests were applied to the data collected.

Results: The rate of noncontributory examinations increased from $43.1 \%$ for the control group to $4.8 \%$ for the new protocol group. The reference enhancement level in the pulmonary trunk is 250 UH. The mean enhancement in the pulmonary trunk of the new protocol group $(332 \mathrm{HU}( \pm 71 \mathrm{HU}( \pm 71 \mathrm{HU}))$ is significantly greater than the reference value of $250 \mathrm{HU}(P<.0001)$, which is not the case for control group $(P=.3485>.05)$, which has a mean enhancement of $239 \mathrm{HU}( \pm 87 \mathrm{HU})$. The control group had a mean DLP of 225 mGy.cm ( $\pm 81 \mathrm{mGy} . \mathrm{cm})$, and the newprotocol group had a mean DLP of 189 mGy.cm $( \pm 75$ mGy.cm).

Discussion: Our noncontributory examination rate is the lowest rate described in the literature. Our protocol contradicts standard practices of placing an ROI in the pulmonary trunk for bolus detection of iodinated contrast media.

Conclusion: The results of this study showed that this protocol reduces the number of noncontributory examinations while reducing the dose delivered to patients. This robust protocol is applicable to other devices and meets perfectly radiation-safety requirements and injected contrast media volume limitation.

\section{RÉSUMÉ}

Introduction : Cette étude présente et évalue un protocole de réalisation d'angioscanner pulmonaire dédié à la femme enceinte. Sa principale particularité consiste à positionner la région d'intérêt (region of interest $\mathrm{ROI}$ ) de détection de bolus de produit de contraste iodé (PCI) directement sur la veine cave supérieure (VCS). L'objectif de ce travail est de démontrer l'efficacité de cette méthode.

Matériel et méthodes : Le protocole consiste à injecter $50 \mathrm{~mL}$ d'un produit de contraste iodé $(350 \mathrm{mgI} / \mathrm{mL})$, à un débit d'injection compris entre 4 et $6 \mathrm{~mL} / \mathrm{sec}$ suivi de $40 \mathrm{~mL}$ de $\mathrm{NaCl}$. La ROI est positionnée sur la veine cave supérieure, le seuil de déclenchement est de $100 \mathrm{UH}$, l'acquisition est réalisée à $100 \mathrm{kVp}$ avec une apnée simple. Ce protocole a été évalué rétrospectivement sur une population de 105 femmes (groupe 1) et comparé avec un groupe témoin (groupe 2) n'ayant pas bénéficié de ce protocole $(\mathrm{n}=55)$. Les deux groupes ont été étudiés sur le même appareil dans le même centre. Chaque examen a bénéficié d'une évaluation permettant de les classer en 3 groupes : examen optimal, sub-obtimal, et non contributif. Les valeurs des produits dose longueur (PDL) ont également été relevées. Des tests statistiques (comparaison de moyennes) ont été appliqués aux données recueillies. \footnotetext{
France.

* Corresponding author: Alfredo Cantarinha, RT, CHU du Kremlin Bicêtre, Service de radiologie générale, 78 rue du Général Leclerc, 94270 Le Kremlin Bicetre, E-mail address: alfredo.cantarinha@orange.fr (A. Cantarinha). 
Résultats : Le taux d'examens non-contributifs est passé de 43,1\% pour le groupe témoin à $4,8 \%$ pour le groupe ayant bénéficié du nouveau protocole. Le rehaussement de référence dans le tronc pulmonaire est de 250 unités Hounsfield (UH). Le rehaussement moyen dans le tronc pulmonaire du groupe étudié $(332 \mathrm{UH}( \pm 71 \mathrm{UH}))$ est significativement au-dessus de la valeur de référence $(P<, 0001)$, ce qui n'est pas le cas du groupe $2(P=0,3485>0,05)$ qui présente un rehaussement moyen de $239 \mathrm{UH}( \pm 87 \mathrm{UH})$. Le groupe témoin affiche un PDL moyen de $225 \mathrm{mGy} . \mathrm{cm}( \pm 81 \mathrm{mGy} . \mathrm{cm})$ et le groupe étudié un PDL moyen de 189 mGy.cm $( \pm 75$ mGy.cm).

Keywords: Pulmonary embolism; pregnancy; CT pulmonary angiography (CTPA)

Mots clés: Embolie pulmonaire; grossesse; angioscanner; artères pulmonaires

\section{Introduction}

L'embolie pulmonaire (EP) est l'une des causes les plus courantes de décès maternel dans les pays développés [1-4]. Cette complication majeure de la grossesse est responsable de $2 \%$ à $14 \%$ de tous les décès maternels dans le monde [5]. La période du post-partum est la période comportant le risque le plus élevé de thromboembolie veineuse; après une césarienne, le risque est 10 fois plus élevé $[4,6]$. Cette fréquence s'explique par un état d'hypercoagulabilité $[1,2,4]$, pouvant être à l'origine de phlébite des membres inférieurs ou encore d'une embolie pulmonaire. Il s'agit de la troisième cause directe de décès maternels en France et au Royaume Uni [1]. Aux Etats-Unis, l'EP est la cause de mortalité la plus courante pendant la grossesse, représentant $10 \%$ des décès [7]. La prise en charge des patientes dans ce contexte doit être rapide et efficace.

L'angioscanner des artères pulmonaires est l'examen de choix compte tenu de sa disponibilité et de sa capacité à mettre en évidence les thrombis (Figure 1). Egalement, l'angioscanner détecte les diagnostics différentiels [1,3,8,9] comme une surcharge pulmonaire d'origine cardiaque, un foyer de pneumopathie, un épanchement pleural, une péricardite ou encore une dissection aortique. L'injection de produit de contraste est indispensable ; il est possible de
Discussion : Notre taux d'examen non contributifs est le taux le plus bas observé dans la littérature. Notre protocole va à l'encontre des pratiques standards qui consistent à placer un ROI dans le tronc pulmonaire pour la détection du bolus de produit de contraste iodé.

Conclusion : Les résultats de cette étude ont démontré que ce protocole diminue le nombre d'examens non-contibutifs tout en réduisant la dose délivrée aux patientes. Ce protocole est simple à mettre en place, et répond parfaitement aux exigences de radioprotection et de limitation de volume de produit de contraste injecté

réaliser cet examen chez une femme enceinte à n'importe quel moment du terme de la grossesse $[4,10]$.

La réussite de l'examen est évaluée a posteriori par les praticiens en tenant compte des 4 critères de réussite (CR) suivants:

- CR1: rehaussement du tronc pulmonaire supérieur à 250 unités Hounsfield (UH) [8].

- CR2: rehaussement de la circulation artérielle pulmonaire plus élevé que celui de la circulation artérielle aortique (circulation systémique).

- CR3: rehaussement homogène des artères pulmonaires se traduisant en distalités (artères lobaires et segmentaires et sous-segmentaires).

- CR4: absence de flou cinétique au niveau distal (basal notamment) et au niveau du parenchyme pulmonaire.

Un examen est dit « optimal » si les 4 critères de réussite (CR1 à CR4) sont retrouvés (Figure 2). Si l'un ou plusieurs critères de réussite ne sont pas atteint, l'interprétation des images est difficile (Figure 3).

Le taux d'angio-scanners des artères pulmonaires non contributifs est plus élevé chez les femmes enceintes, de 5,6\% à $35,7 \%$ [7,12-14] ; que pour le reste de la population (de $6,4 \%$ à $7,5 \%)$ [8,12]. Par ailleurs, plusieurs publications
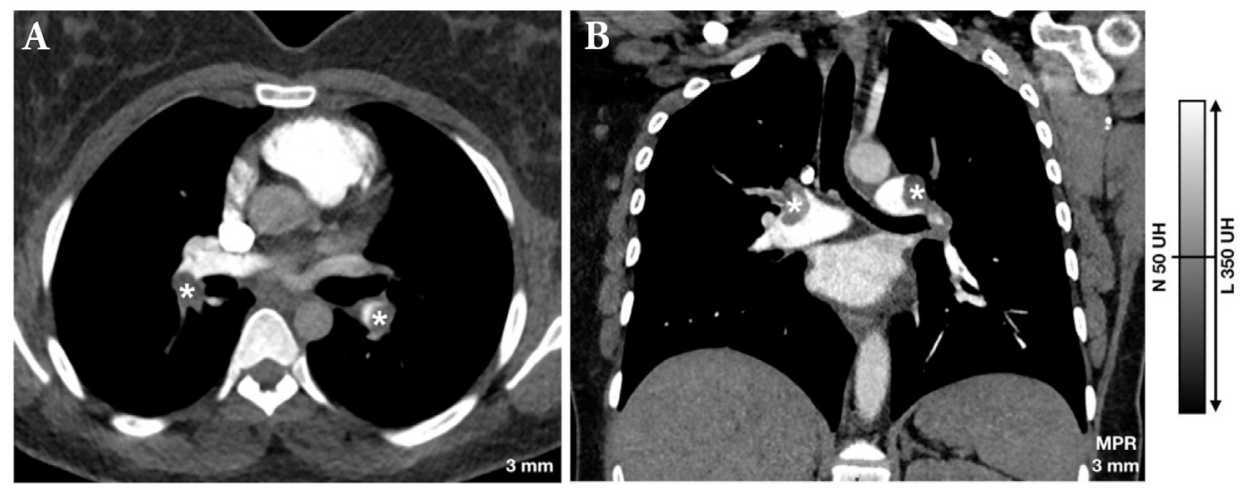

Figure 1. Angioscanners des artères pulmonaires réalisés chez des femmes enceintes, présentant des thrombis $\left(^{*}\right)$. Patiente de 41 ans à $8 \mathrm{SA}(\mathrm{A})$, patiente de 34 ans à 11 SA (B). 

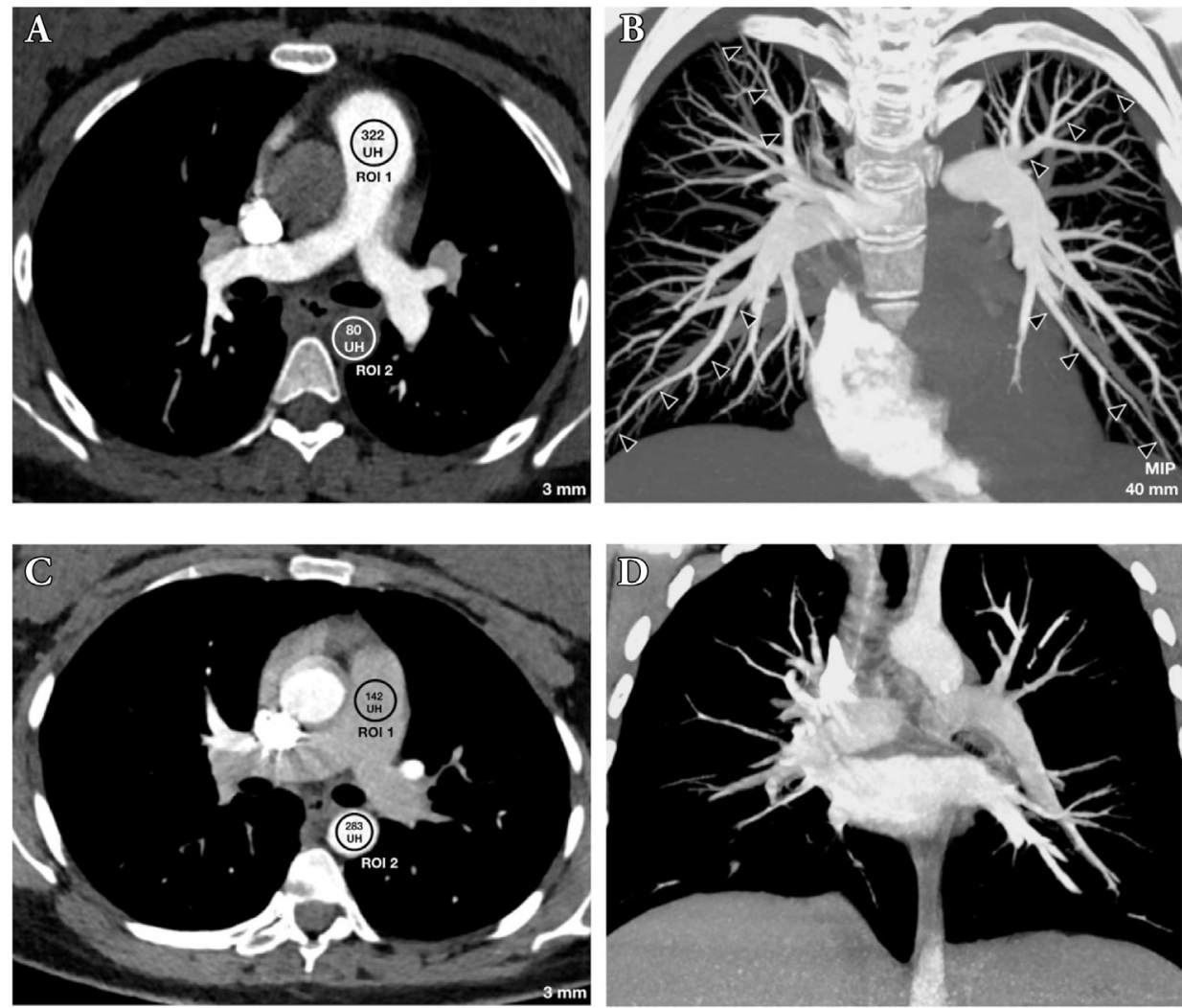

Figure 2. A et B. Respects des critères de réussites (CR) d'un angioscanner pulmonaire présentés sur une coupe transversale passant par le tronc pulmonaire (A), sur une reconstruction MIP coronale passant par les artères pulmoniares droite et gauche (B). CR1: Le rehaussement du tronc pulmonaire est supérieur à 250 UH (ROI 1). CR2: le rehaussement artériel pulmonaire est supérieur au rehaussement aortique (ROI $1>$ ROI 2). CR3: le rehaussement pulmonaire apparait homogène et se traduit en distalité (têtes de flèches noires). CR4: absence de flou cinétique gênant. L'examen présenté ci-dessus est ainsi qualifié d' " optimal ». C et D. Nonobtention des critères de réussites d'un angioscanner pulmonaire présentés sur une coupe transversale passant par le tronc pulmonaire (C), sur une reconstruction MIP coronale passant par les artères pulmonaires droite et gauche (D). Non-obtension du CR1: le rehaussement de tronc pulmonaire est inférieur à $250 \mathrm{UH}$ (ROI 1). Non-obtention du CR2: le rehaussement artériel pulmonaire est inférieur au rehaussement aortique (ROI $1<$ ROI 2 ). La non-obtention des critères 1 et 2 rend difficile l'analyse du réseau artériel pulmonaire (CR3) même en l'absence de flou cinétique gênant (validation du CR4). L'examen présenté ci-dessus est qualifié de «non contributif ».

évoquent ou rapportent la qualité moindre de ces examens (= examens sous optimaux) réalisés en cours de grossesse $[1,7,12-17]$, sans citer systématiquement des chiffres.

Ce taux d'examens sous optimaux ou non contributifs plus élevés chez la femme enceinte s'explique principalement par les modifications physiologiques occasionnées par la grossesse, modifications que l'on retrouve jusqu'à plusieurs semaines en post-partum [18]. La grossesse occasionne une augmentation de la fréquence cardiaque (10 à 20 battements/min), du débit cardiaque (30\% à $50 \%)$ et de la volémie $(30 \%$ à $50 \%)$ [7,18,19]. Ces modifications physiologiques peuvent engendrer une dilution hémodynamique du produit de contraste $[7,13,16,17]$ et être ainsi responsable d'une opacification insuffisante des artères pulmonaires (Figure 2), qui rend difficile l'interprétation des images.

Les facteurs influençant la qualité du rehaussement artériel pulmonaire sont donc nombreux et peuvent être répartis en trois groupes (Figure 3) [11] :
- les facteurs liés à la patiente, avec les spécificités physiologiques de la grossesse et du post partum (augmentation du débit et du rythme cardiaque, augmentation de la volémie) ainsi que son poids et sa taille.

- les facteurs liés au protocole d'injection de produit de contraste (volume, débit et concentration en iode, mais également la qualité de la voie d'abord).

- les facteurs liés au protocole d'acquisition du scanner (durée d'acquisition, délai et vitesse de déplacement de la table d'examen, tension).

Le nombre importants d'examens non-contributifs retrouvés dans nos pratiques et dans la littérature [12-14] nous a amené à modifier nos pratiques en proposant un nouveau protocole.

Cet article présente le développement d'un protocole de prise en charge de la femme enceinte pour la réalisation d'un angioscanner des artères pulmonaires permettant potentiellement de limiter le taux d'examens sub-optimaux (= examen interprétable mais sans respect de tous les critères CR1 à CR4) ou non contributifs (= interprétation difficile 


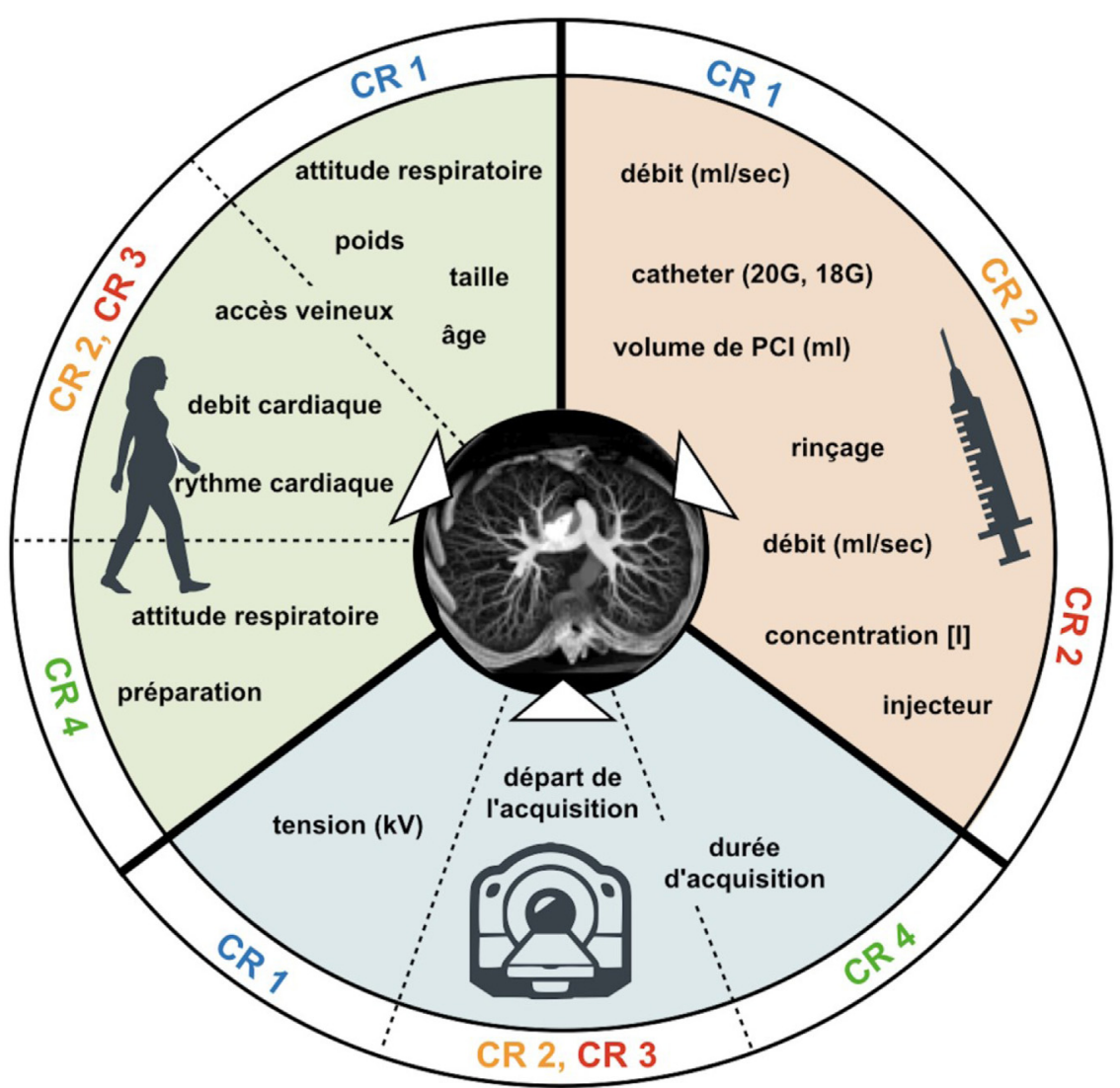

Figure 3. Répartition en 3 groupes (patiente, acquisition, injection) des facteurs impactant l'obtension des 4 critères de réussite (CR1, CR2, CR3, CR4) d'un angioscanner artériel pulmonaire chez la femme enceinte [11].

voire impossible) qui sont difficiles à interpréter, voire ininterprétables. Une compréhension approfondie de la dynamique du produit de contraste est essentielle à la conception d'un protocole [11] ; c'est pourquoi la conception de ce protocole tient compte de l'ensemble des facteurs cités en sus (Figure 3). Il a pour objectif d'être transposable à la majorité des appareils actuels, car cette démarche est basée sur la compréhension de la dynamique du produit de contraste et la nécessité de s'adapter aux spécificités des patientes.

Une comparaison rétrospective entre deux populations de patientes (groupe avant et groupe après mise en place du protocole spécifique) sera présentée et permettra d'évaluer l'intérêt de ce protocole dédié à la femme enceinte, en tenant compte de critères d'évaluations objectifs. Les bilans dosimétriques accompagneront également la présentation de nos résultats.

\section{Matériel et Méthode}

\section{Considérations Éthiques}

L'étude présentée dans cet article est rétrospective. Elle a donc été effectuée sans être évaluée par le comité d'éthique de l'établissement. Aucun consentement écrit n'a été requis étant donné que les acquisitions ont été effectuées conformément au protocole de routine validé par le service. Les informations concernant l'identité des patients ont été supprimées avant analyse. L'ensemble des dossiers ont été récupérés via le système d'archivage (Picture Archiving and Communication System: PACS) de l'établissement (PACS Carestream, Version 12.1.5.1046 - Rochester, État de New York, ÉtatsUnis).

\section{Protocole d'angioscanner Pulmonaire Spécifique à la Femme Enceinte}

Notre travail d'optimisation s'est inspiré des conclusions de travaux parus dans la littérature scientifique et dont nous avons décidé de retenir 4 axes principaux pour le développement de notre protocole :

1 L'acquisition plus précoce [12].

2 L'augmentation du débit d'injection [7,17].

$3 \mathrm{La}$ proscription de l'inspiration profonde $[7,12,13,17,20,21]$.

4 Privilégier la tension à $100 \mathrm{kVp}$ [17,22,23].

\section{Produit de Contraste Iodés (PCI) et Paramètres d'injection}

L'ensemble des examens ont été réalisés avec une concentration en iode de $350 \mathrm{mgI} / \mathrm{mL}$. Deux PCI ont été utilisés, Optiject 350 (Ioversol - Guerbet, Villepinte, France) et Ioméron 350 (Ioméprol - Bracco Imaging, Courcouronnes, France). La possibilité de 2 options permet une alternative en cas de réaction allergique connue à l'une des 2 molécules (Ioversol, Ioméprol). 
Toutes les acquisitions ont été réalisées avec un injecteur automatique à double tête Optivantage DH (Guerbet, Villepinte, France). Le volume de PCI était fixé à $50 \mathrm{~mL}$ suivi d'un rinçage $(\mathrm{NaCl})$ de $40 \mathrm{~mL}$. Le débit d'injection (PCI/ $\mathrm{NaCl}$ ) était compris entre 4 et $6 \mathrm{~mL} / \mathrm{s}$ en privilégiant dès que possible le débit le plus élevé.

La mise en place d'une voie veineuse de qualité était réalisée avec un cathéter court de 18 Gauge $(\mathrm{G})$ dans la région de la fosse cubitale (" pli du coude »).

\section{Consignes Respiratoires}

Les acquisitions ont systématiquement été réalisées en apnée simple pour limiter le risque de phénomène de Valsalva (Note sur la manœuvre de "Valsalva »: lorsque la patiente prend une inspiration profonde et " pousse » sur les muscles abdominaux au moment de l'acquisition, cela provoque un afflux de sang " non iodé 》 véhiculé par la veine cave inférieure dans l'atrium droit (phénomène de Valsalva), qui diluera presque totalement le contraste provenant de la veine cave supérieure. L'apport de la veine cave inférieure « prend le dessus » sur celui de la veine cave supérieure créant une " interruption transitoire du contraste " émanant de cette dernière. Par conséquence, le rehaussement des artères pulmonaires sera moindre, voire inexistant du fait de cette dilution hémodynamique. Le raisonnement est donc de proscrire l'inspiration profonde et de demander une simple apnée lors de l'acquisition). Ce dernier pouvant accentuer la dilution du PCI dans l'atrium droit et provoquer par conséquent une baisse de rehaussement des artères pulmonaires [7,20,21].

L'apnée simple, nécessaire à la bonne opacification des artères pulmonaires, limite cependant l'analyse $\mathrm{du}$ parenchyme pulmonaire qui est facilité avec une inspiration profonde [24].

\section{Appareil, Paramètres et Protocole d'acquisition}

Les acquisitions ont été réalisées sur un scanner multicoupes (Definition AS+, Siemens, Erlangen, Germany), fonctionnant en mode hélicoïdal. L'acquisition est mono énergétique avec une tension de $100 \mathrm{kVp}$ afin de se rapprocher du pic d'absorption de l'iode pour améliorer le rehaussement de contraste [25]. D'autre part cette tension permet (par rapport aux protocoles calibrés à $120 \mathrm{kVp}$ ) de réduire la dose délivrée [22,23]. L'ampérage est modulé automatiquement par l'appareil (CARE Dose 4D, Siemens) [26]. Les images sont reconstruites par un algorithme itératif appliqué aux données brutes (Safire, Siemens) [27-29].

Pour les paramètres programmés (collimation: $38,4 \mathrm{~mm}$; Pitch: 1,4; vitesse d'avancée de la table $10,7 \mathrm{~cm} / \mathrm{sec}$ ) ; les durées moyennes permettant l'acquisition d'un thorax complet (de 20 à $30 \mathrm{~cm}$ selon la taille de la patiente) varient de 3 à 4 secondes.

Le déclenchement de l'acquisition se réalise lorsque la région d'intérêt (ROI) placée dans la veine cave supérieure (coupe transversale à hauteur de la vertèbre T6) atteint le seuil de $100 \mathrm{UH}$ (Figure 4). Ce choix permet de tenir compte des durées incompressibles de déplacement de la table faisant suite au déclenchement de l'acquisition ( 5 secondes) et de l'acquisition proprement dite (3 à 4 secondes) (Figure 4).

\section{Analyse Rétrospective}

Le protocole proposé dans le paragraphe précédent (2.1) a été mis en place en juin 2015 dans notre unité. Afin d'évaluer

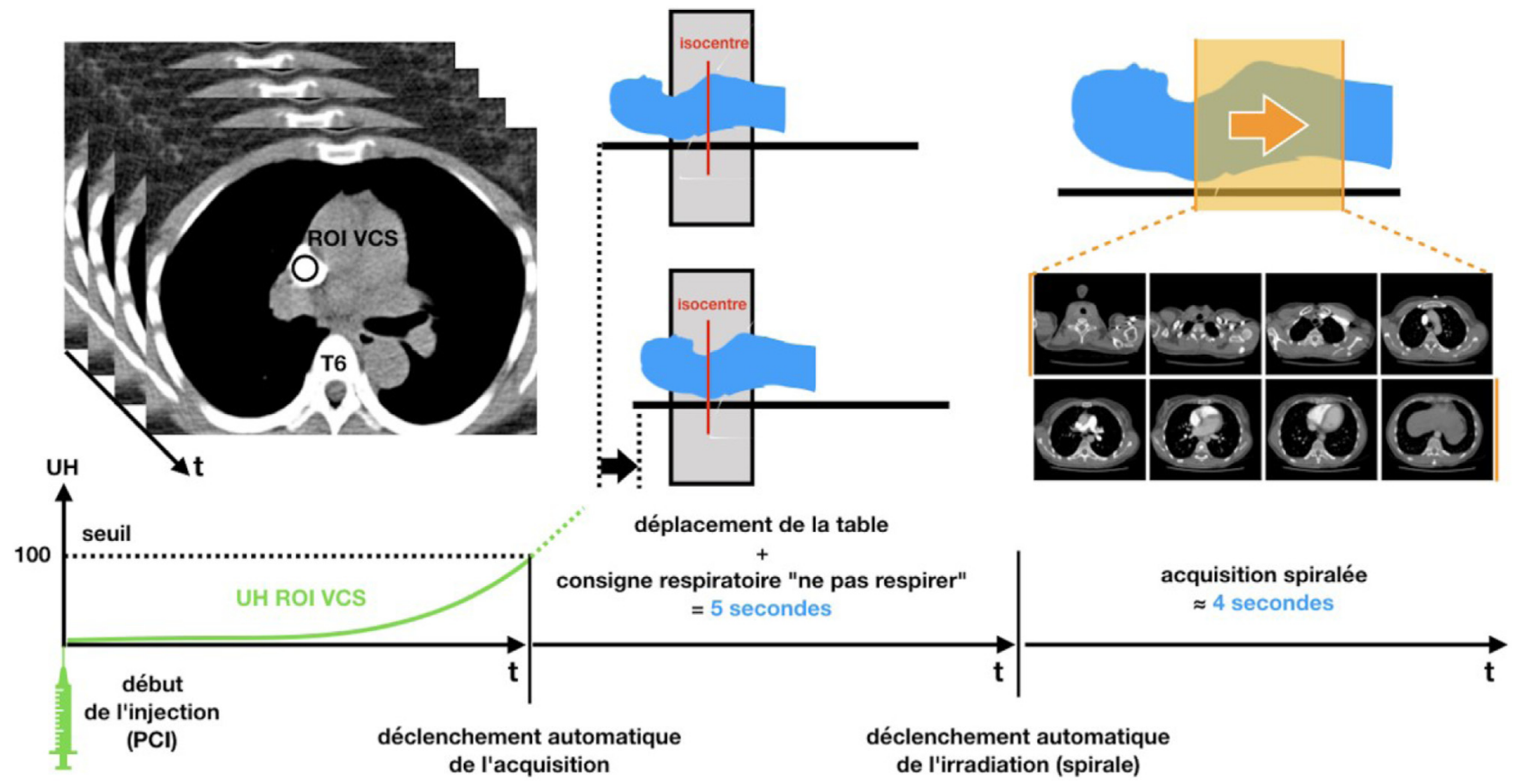

Figure 4. Les différentes étapes du protocole spécifique; du début de l’injection à la fin de l'exposition. L'acquisition se déclenche lorsque la ROI placée dans la VCS atteint le seuil de $100 \mathrm{UH}$; un délai incompressible de 5 secondes permet de positionner la patiente au point de départ et d'appliquer les consignes d'attitude respiratoire; l'acquisition proprement dite s'effectue en 3 secondes environs. 
son efficacité une analyse rétrospective a été effectuée sur 2 groupes de patientes enceintes ayant bénéficié d'un angioscanner des artères pulmonaires pour recherche d'EP, ce qui correspondait à l'unique critère d'inclusion.

- Groupe 1 : avec protocole spécifique appliqué systématiquement (de juin 2015 à aout 2019).

- Groupe 2 (témoin) : sans protocole spécifique (de décembre 2009 à mai 2015). Le protocole non spécifique différait de celui développé dans cette étude par une valeur de tension à $120 \mathrm{kVp}$, la mise en place d'un ROI sur le tronc pulmonaire (seuil à $100 \mathrm{UH}$ ), l'absence de consensus concernant l'injection (concentration, débit, volume) et absence de précautions particulière concernant le choix du calibre du cathéter et du site d'injection (région du " pli du coude », du « dos de la main »,...).

Les acquisitions des 2 groupes ont été évaluées selon les critères (CR1 à CR4) illustrés sur la Figure 2, puis classées en 3 catégories :

- Examen optimal (en cas de respect des 4 critères).

- Examen sub-optimal (en cas d'examen interprétable mais sans respect de tous les critères).

- Examen non contributif (en cas d'interprétation difficile voire impossible).

Un relevé des PDL a également été réalisé dans les deux groupes.

\section{Analyses Statistiques}

La population des 2 groupes a été répartie en 4 sousgroupes en fonction du stade de grossesse:

- Sous-groupe semestre 1 (de 0 à $13 \mathrm{SA}$ )

- Sous-groupe semestre 2 (14 à $26 \mathrm{SA}$ )

- Sous-groupe semestre 3 (de 27 à 40 SA)

- Sous-groupe post-partum

Un test exact de Fisher a été appliqué pour vérifier s'il n'existe pas de différences significatives de répartition semestrielle entre les 2 groupes.
Pour établir si les différences des données quantitatives (UH, PDL) sont significatives entre les 2 groupes, des tests statistiques (comparaisons de moyennes) ont été réalisés en utilisant le t-test de Student (S) ou le test de Mann-Whitney Wilcoxon (W) quand la distribution des variables ne suivait pas une distribution normale (i.e. test de Student non applicable). La distribution des variables a été analysée graphiquement à l'aide d'histogrammes pour évaluer la normalité.

Une formulation bilatérale a été choisie pour tous les tests, et une valeur $P<0,05$ (risque alpha de 5\%) est considérée comme statistiquement significative. Ces analyses ont été conduites avec le logiciel Statistical Analysis System 9.4 (SAS Institute, Cary, NC).

\section{Résultats}

Présentation des résultats des 2 Groupes

Les résultats des groupe 1 et 2 sont présentés dans le Tableau 1 et la Figure 5.

Le rehaussement artériel pulmonaire moyen est plus élevé de $38 \%$ dans le groupe 1 (332 UH) par rapport au groupe 2 (239 UH) (Tableau 1, Figure 5B).

Le PDL moyen est plus faible de $-18 \%$ dans le groupe 1 (189 mGy.cm) par rapport au groupe 2 (225 mGy.cm) (Tableau 1, Figure 5C).

\section{Comparaisons Statistiques des 2 Groupes (UH, PDL)}

Le test exact de Fisher appliqué comparant la répartition en sous-groupe de chaque groupe présente une valeur $P$ égale à 0,5157 , ce qui signifie qu'il n'existe pas de différence significative entre les deux groupes.

La comparaison des moyennes concernant le rehaussement (UH) du tronc pulmonaire réalisée avec un t-test de Student, montre que les 2 groupes sont significativement différents : $P$ $<0,0001$. Le rehaussement moyen du groupe 1 est significativement au-dessus de la valeur de référence de 250UH $(P<$ $0,0001)$, ce qui n'est pas le cas du groupe $2(P=0,3485>$ $0,05)$.

La comparaison des PDL des deux groupes s'est effectuée avec un test de Mann-Whitney Wilcoxon (W) et montre

Tableau 1

Présentation des résultats des Groupes 1 et 2

\begin{tabular}{|c|c|c|}
\hline & $\begin{array}{l}\text { Groupe } 1 \text { (nouveau protocole) } \\
\text { de juin } 2015 \text { à aout } 2019\end{array}$ & $\begin{array}{l}\text { Groupe } 2 \text { (témoin) de décembre } \\
2009 \text { à mai } 2015\end{array}$ \\
\hline Nombre d'examens & 105 & 58 \\
\hline âge moyen des patientes ( \pm écart-type) (Figure $5 \mathrm{~A}$ ) & 30,9 ans ( $\pm 5,3$ ans) & 28,4 ans ( $\pm 4,4$ ans) \\
\hline \multirow{3}{*}{ répartition des patientes (stade de grossesse) } & $2^{\mathrm{e}}$ trimestre $19,0 \%$ & $2^{\mathrm{e}}$ trimestre $19,0 \%$ \\
\hline & $3^{\mathrm{e}}$ trimestre $48,6 \%$ & $3^{\mathrm{e}}$ trimestre $37,9 \%$ \\
\hline & post-partum $27,6 \%$ & post-partum $36,2 \%$ \\
\hline le PDL moyen (土écart-type) (Figure 5C) & 189 mGy cm $( \pm 75$ mGy.cm $)$ & 225 mGy cm $( \pm 81$ mGy.cm $)$ \\
\hline \multirow[t]{3}{*}{ Classification des examesns } & optimal : $64,8 \%(n=68)$ & optimal : 22,4\% (n = 13) \\
\hline & sub-optimal : $30,4 \%(n=32)$ & sub-optimal : $34,5 \%(n=20)$ \\
\hline & non-contributifs : $4,8 \%(\mathrm{n}=5)$ & non-contributifs : $43,1 \%(n=25)$ \\
\hline
\end{tabular}




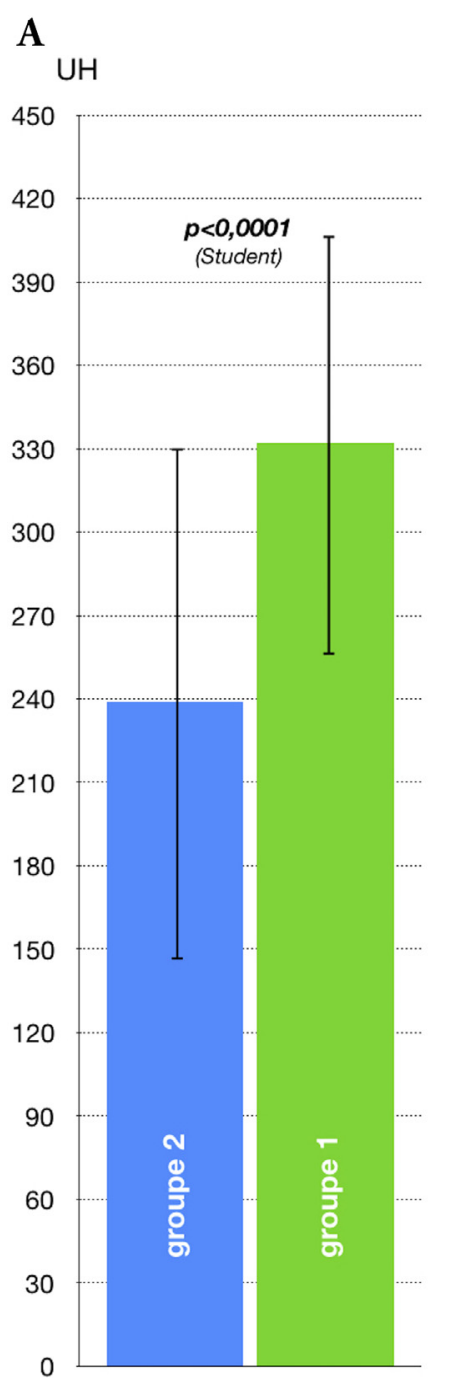

Rehaussement
B

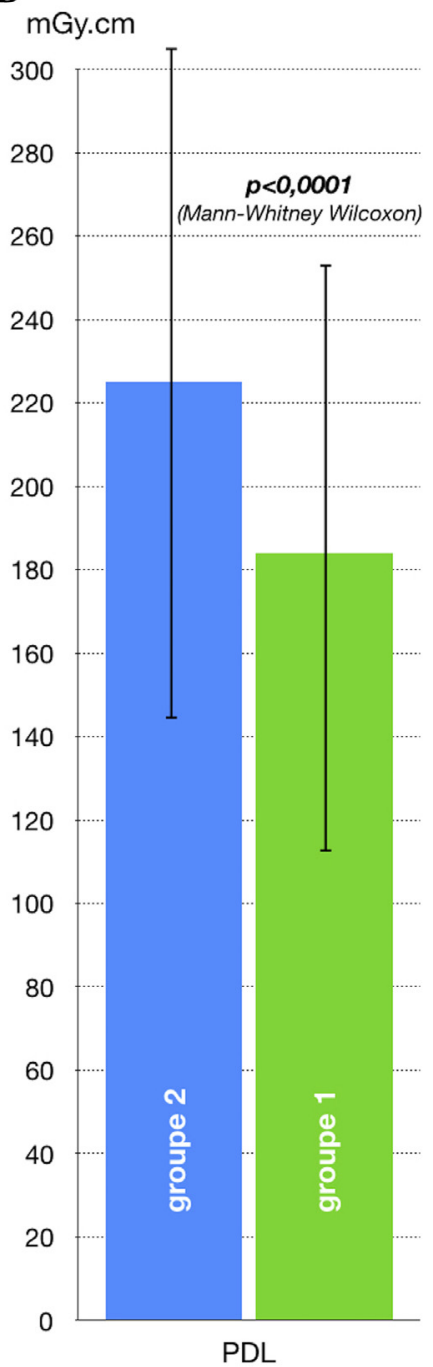

C

évaluation

Optimal

sub-optimal

non-contributif
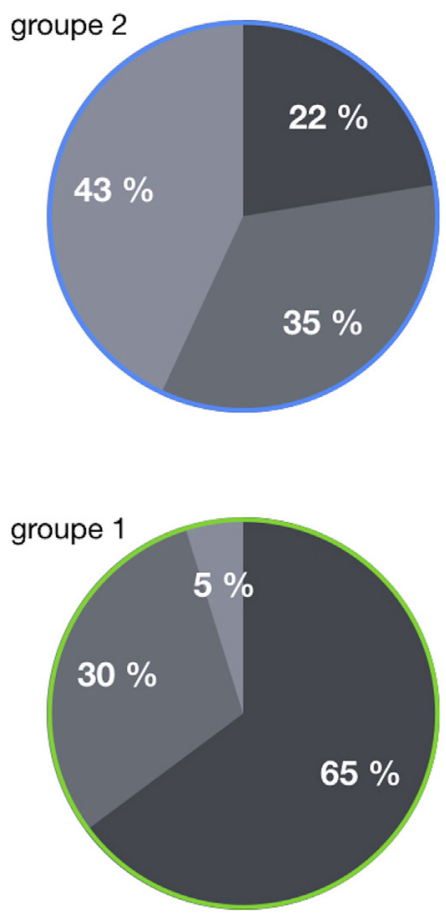

Figure 5. Résutats en provenance des deux groupes 2 (protocole non spécifique) et 1 (protocole spécifique) concernant le rehaussement artériel pulmonaire (A), le PDL (B), la classification des examens (C).

que les 2 groupes sont significativement différents $(P<$ $0,0001)$.

\section{Discussions}

\section{Analyse des résultats}

Nos résultats montrent une amélioration significative des examens en termes d'opacification $(P<0,0001)$ et de réussite pour le groupe ayant bénéficié du protocole spécifique. Le taux d'examens non contributifs est passé sous la barre des $5 \%$ pour une population importante (105 examens) ce qui présente à notre connaissance le taux le plus bas décrit dans la littérature qui selon les articles se situe entre 5,6\% et $35,7 \%[7,12-14]$ chez la femme enceinte. Notre taux est même inférieur au taux d'examens non contributifs pour la population générale (de 6,4 à 7,5\%) [8,12].
Au niveau du PDL, une diminution significative $(P<$ 0,0001) a également été observée entre les 2 groupes (de $225 \mathrm{mGy} . \mathrm{cm}$ à $189 \mathrm{mGy} . \mathrm{cm}$ ) ce qui s'explique principalement par l'usage systématique d'une tension de $100 \mathrm{kVp}$ $[22,23]$.

\section{Transferabilité du Protocole à d'autres Appareils}

La question concernant la transférabilité de ce protocole (donc des résultats) à d'autres appareils doit être discutée. La mise en place de notre protocole doit respecter systématiquement les points suivants :

- Préparation de l'injection : PCI à $350 \mathrm{mgI} / \mathrm{mL} ; 50 \mathrm{~mL}$ (PCI); $40 \mathrm{~mL}(\mathrm{NaCl}) ; 4$ à $6 \mathrm{~mL} / \mathrm{sec}$; cathéter de 18G.

- Détection du bolus : ROI sur veine cave supérieure $(+++)$, seuil de déclenchement de $100 \mathrm{UH}$. 
Tableau 2

Résultats de l'application du Protocole Spécifique Obtenus sur Différents Appareils (scanner1 et Scanner 2)

\begin{tabular}{|c|c|c|c|}
\hline \multirow[t]{2}{*}{ Modèle/marque } & Scanner de référence (étude) & Scanner 1 & Scanner 2 \\
\hline & Definition AS+/Siemens & Revolution Discovery HD/GEMS & Somatom Drive/Siemens \\
\hline Nombre d'examens réalisés & $\mathrm{n}=105($ groupe 1$)$ & $\mathrm{n}=6$ & $\mathrm{n}=7$ \\
\hline $\begin{array}{l}\text { Rehaussement du tronc pulmonaire en UH } \\
\text { (moyenne } \pm \text { écart-type) }\end{array}$ & $331( \pm 75) \mathrm{UH}$ & $366( \pm 110) \mathrm{UH}$ & 415 (土94) UH \\
\hline PDL en mGy.cm (moyenne \pm écart-type) & $189( \pm 75) \mathrm{mGy} \cdot \mathrm{cm}$ & $260( \pm 80)$ mGy.cm & $94( \pm 71) \mathrm{mGy} \cdot \mathrm{cm}$ \\
\hline $\begin{array}{l}\text { \%age d'examen optimal, sub-obtimal, } \\
\text { non-contributif }\end{array}$ & $65 \%, 30 \%, 5 \%$ & $83 \%, 17 \%, 0 \%$ & $100 \%, 0 \%, 0 \%$ \\
\hline
\end{tabular}

- Acquisition: démarre 5 secondes après l'atteinte du seuil (100 UH), apnée simple (pas d'inspiration profonde), $100 \mathrm{kVp}$, durée d'acquisition de 3 à 4 sec.

Ces points sont a priori transférables sur tous les modèles de scanners de génération équivalente voire plus récents. Le seul point pouvant être plus complexe à mettre en place concerne la durée d'acquisition qui est multiparamétrique (nombre de barrettes en jeu, vitesse de rotation, pitch, ...). Néanmoins, quel que soit la configuration technologique utilisée, programmer une durée d'acquisition de l'ordre de 3 à $4 \mathrm{sec}$ est possible sur la plupart des appareils. A titre d'exemple nous avons expérimenté ce protocole sur un nombre faible $(\mathrm{n}=7)$ de patientes sur deux autres appareils différents et plus réc. Les données présentées dans le Tableau 2 montrent que l'application de notre protocole sur deux autres appareils présente des résultats équivalents voire meilleurs que ceux obtenus dans le groupe 2 en termes de rehaussement (UH) et de réussite ce qui semble confirmer la robustesse du protocole proposé dans notre étude. Les données présentées dans le Tableau 2 semblent même suggérer que des améliorations ont encore possibles pour certains appareils (scanner 2) en termes de rehaussement et de réduction de dose et qui pourront faire l'objet d'évaluations complémentaires.

\section{Comparaison avec d'autres Protocoles Scanographiques}

D'autres protocoles spécifiques existent dans la littérature et sont utilisés en pratique clinique. Nous allons dans ce qui suit comparer notre méthode aux autres protocoles connus.

\section{Protocoles « Standards》}

Ces protocoles consistent à placer le ROI de détection de l'arrivée du PCI dans l'atrium droit ou le tronc pulmonaire avec un seuil prédéfini de 100 à $150 \mathrm{UH}$. Ce type de protocoles (qui correspond au protocole du groupe 2 de notre étude), est très utilisé en routine clinique et est bien décrit dans la littérature $[8,13,24,30]$. Notre étude, qui propose de positionner la ROI dans la VCI, va ainsi à l'encontre de ce dogme de mise en place de la ROI dans le tronc pulmonaire ou l'atrium droit. Néanmoins les résultats de notre étude démontrent que notre protocole (Figure 4) est pertinent et judicieux au regard du faible taux d'échecs observés (Figure 5 et Tableau 1) par rapport aux données de la littérature $(4,8 \%$ pour notre protocole vs $5,6 \%$ à $35,7 \%$ [7,12-14]).

\section{Protocoles à « Test-bolus »}

Protocole utilisant un « test-bolus » qui utilise une première injection de PCI (de 5 à $20 \mathrm{~mL}$ ), permettant d'évaluer le temps mis par ce « volume test » pour atteindre les artères pulmonaires (time to peak-TTP) et fixer ainsi le délai d'acquisition pour la deuxième injection qui correspondra à l'acquisition effective [24,31]. En termes de qualité de rehaussement, les résultats proposés par ce protocole semblent être équivalents au nôtre. Néanmoins aucune étude n'a évalué les taux de réussites/échecs avec ce protocole. Egalement, en pratique, ce protocole demande plus de temps que le nôtre du fait de la réinjection nécessaire au calcule du TTP. L'étude de Saade [31] met en avant une baisse significative du volume de PCI injecté qui se situe à $33 \pm 9 \mathrm{~mL}$ ce qui se situe juste à peine en-dessous de notre volume fixé à $50 \mathrm{~mL}$. Au regard des résultats présentés dans le Tableau 2, une diminution du volume de PCI injecté semble être envisageable dans une démarche d'optimisation.

\section{Protocoles d'imagerie Spectrale}

Protocole utilisant la double énergie. L'usage de l'imagerie spectrale semble une alternative intéressante dans ce contexte pour permettre de compenser une acquisition non optimale notamment en reconstruisant des séries mono énergétiques à $45 \mathrm{keV}$ [30]. De plus, l'imagerie spectrale permet de reconstruire une cartographie d'iode permettant d'accéder à une analyse quantitative de perfusion mais à condition d'avoir une acquisition longue $(\approx 7 \mathrm{sec}$ ) qui impacte la qualité des images UH traditionnelles [32,33]. Cependant cette imagerie n'est pas encore disponible sur tous les appareils et expose à un surcroît d'irradiation compris entre $5 \%$ et $40 \%$ $[30,34,35]$.

\section{Conclusion}

Le protocole présenté dans cet article présente des taux de réussite élevés par rapport aux protocoles standards et aux données retrouvées de la littérature. Ce protocole performant est applicable à d'autres appareils, simple à mettre en place, et répond parfaitement aux exigences de radioprotection et de limitation de volume de produit de contraste injecté. L'utilisation de ce protocole est recommandé lors de la réalisation d'angioscanner pulmonaires chez la femme enceinte. Ce 
travail s'inscrit parfaitement dans la démarche de pratiques basées sur des preuves (evidence based pratice: EBP).

\section{Remerciements}

Les auteurs tiennent à remercier l'équipe médicale et paramédicale du service de radiologie générale de l'hôpital Bicêtre. Ils remercient également le Dr Clément Palpacuer pour son aide concernant l'analyse statistique des données.

\section{References}

[1] Lonjaret L, Lairez O, Minville V, Bayoumeu F, Fourcade O, Mercier FJ. Pulmonary embolism and pregnancy. Ann Fr Anesth Reanim. 2013;32(4):257-266.

[2] Bourjeily G, Paidas M, Khalil H, Rosene-Montella K, Rodger M. Pulmonary embolism in pregnancy. Lancet. 2010;375(9713):500-512.

[3] Renner J, Mercier FJ, Le CNEMM. Study of the 21 cases of thromboembolism from the 4th report of national confidential enquiry into maternal death in France in 2007-2009. J Gynecol Obstet Biol Reprod. 2016;45(6):608-618.

[4] Parent F, Jovan R, Colas des Francs V. Venous thromboembolism during pregnancy. Rev Prat. 2015;65(2):188-192.

[5] Tromeur C, van der Pol LM, Le Roux PY, et al. Computed tomography pulmonary angiography versus ventilation-perfusion lung scanning for diagnosing pulmonary embolism during pregnancy: a systematic review and meta-analysis. Haematologica. 2019;104(1):176-188.

[6] Heit JA, Kobbervig CE, James AH, Petterson TM, Bailey KR, Melton 3rd LJ. Trends in the incidence of venous thromboembolism during pregnancy or postpartum: a 30-year population-based study. Ann Intern Med. 2005;143(10):697-706.

[7] Ridge CA, Mhuircheartaigh JN, Dodd JD, Skehan SJ. Pulmonary CT angiography protocol adapted to the hemodynamic effects of pregnancy. AJR Am J Roentgenol. 2011;197(5):1058-1063.

[8] Jolibert M, Vidal V, Cohen F, et al. Improvement of pulmonary angiography in the framework of a maintenance of certification. J Radiol. 2011;92(1):20-24.

[9] Gut-Gobert C, Couturaud F, Leroyer C, Sanchez O. Prise en charge de l'embolie pulmonaire aiguë. Revue de Pneumologie Clinique. 2008;64:298-304.

[10] Sadro CT, Dubinsky TJ. CT in pregnancy: risks and benefits. Appl Radiol. 2013;42:6-16.

[11] Weininger M, Michael Barraza J, Kemper CA, Kalafut JF, Costello P, Joseph Schoepf U. Cardiothoracic CT angiography: current contrast medium delivery strategies. AJR Am J Roentgenol. 2011;196(3):W260W272.

[12] U-King-Im JM, Freeman SJ, Boylan T, Cheow HK. Quality of CT pulmonary angiography for suspected pulmonary embolus in pregnancy. Eur Radiol. 2008;18(12):2709-2715.

[13] Revel MP, Cohen S, Sanchez O, et al. Pulmonary embolism during pregnancy: diagnosis with lung scintigraphy or CT angiography? Radiology. 2011;258(2):590-598.

[14] Chan WS, Rey E, Kent NE. Venous thromboembolism and antithrombotic therapy in pregnancy. J Obstet Gynaecol Can. 2014;36(6):527-553.

[15] Jordan EJ, Godelman A, Levsky JM, Zalta B, Haramati LB. CT pulmonary angiography in pregnant and postpartum women: low yield, high dose. Clin Imaging. 2015;39(2):251-253.

[16] Andreou AK, Curtin JJ, Wilde S, Clark A. Does pregnancy affect vascular enhancement in patients undergoing CT pulmonary angiography? Eur Radiol. 2008;18(12):2716-2722.
[17] Schaefer-Prokop C, Prokop M. CTPA for the diagnosis of acute pulmonary embolism during pregnancy. Eur Radiol. 2008;18(12):2705-2708.

[18] Abbas AE, Lester SJ, Connolly H. Pregnancy and the cardiovascular system. Int J Cardiol. 2005;98(2):179-189.

[19] Adam K. Pregnancy in women with cardiovascular diseases. Methodist Debakey Cardiovasc J. 2017;13(4):209-215.

[20] Kuzo RS, Pooley RA, Crook JE, Heckman MG, Gerber TC. Measurement of caval blood flow with mri during respiratory maneuvers: implications for vascular contrast opacification on pulmonary CT angiographic studies. AJR Am J Roentgenol. 2007;188(3):839-842.

[21] Conrad W, Albert J, Yoo MD. Transient interruption of contrast on CT pulmonary angiography proof of mechanism. J Thorac Imaging. 2007;22(2):125-129.

[22] Heyer CM, Mohr PS, Lemburg SP, Peters SA, Nicolas V. Image quality and radiation exposure at pulmonary CT angiography with 100- or 120 $\mathrm{kVp}$ protocol : prospective randomized study. Radiology. 2007:245(2):577-583.

[23] Feuchtner GM, Jodocy D, Klauser A, et al. Radiation dose reduction by using $100-\mathrm{kV}$ tube voltage in cardiac 64-slice computed tomography: a comparative study. Eur J Radiol. 2010;75(1):e51-e56.

[24] Moore AJ, Wachsmann J, Chamarthy MR, Panjikaran L, Tanabe Y, Rajiah P. Imaging of acute pulmonary embolism: an update. Cardiovasc Diagn Ther. 2018;8(3):225.

[25] Abudurexiti A, Kameda M, Sato E, et al. Demonstration of iodine Kedge imaging by use of an energy-discrimination X-ray computed tomography system with a cadmium telluride detector. Radiol Phys Technol. 2010;3(2):127-135.

[26] Söderberg M. Overview, practical tips and potential pitfalls of using automatic exposure control in CT: Siemens CARE Dose 4D. Radiat Prot Dosimetry. 2016;169(1-4):84-91.

[27] Padole A, Ali Khawaja RD, Kalra MK, Singh S. CT radiation dose and iterative reconstruction techniques. AJR Am J Roentgenol. 2015;204(4):W384-W392.

[28] Scharf M, Brendel S, Melzer K, et al. Image quality, diagnostic accuracy, and potential for radiation dose reduction in thoracoabdominal CT, using Sinogram Affirmed Iterative Reconstruction (SAFIRE) technique in a longitudinal study. PLoS One. 2017;12(7):e0180302.

[29] Pontana F, Moureau D, Schmidt B, et al. CT pulmonary angiogram with $60 \%$ dose reduction: Influence of iterative reconstructions on image quality. Diagn Interv Imaging. 2015;96(5):487-493.

[30] Ohana M, Jeung MY, Labani A, El Ghannudi S, Roy C. Thoracic dual energy CT: acquisition protocols, current applications and future developments. Diagn Interv Imaging. 2014;95(11):1017-1026.

[31] Saade C, Mayat A, El-Merhi F. Exponentially decelerated contrast media injection rate combined with a novel patient-specific contrast formula reduces contrast volume administration and radiation dose during computed tomography pulmonary angiography. J Comput Assist Tomogr. 2016;40:370-374.

[32] Lu GM, Zhao Y, Zhang LJ, Schoepf UJ. Dual-energy CT of the lung. AJR Am J Roentgenol. 2012;199(5 Suppl):S40-S53.

[33] Murphy A, Cheng J, Pratap J, Redman R, Coucher J. Dual-energy computed tomography pulmonary angiography: comparison of vessel enhancement between linear blended and virtual monoenergetic reconstruction techniques. J Med Imaging Radiat Sci. 2019;50(1):62-67.

[34] Remy-Jardin M, Faivre JB, Pontana F, Remy J. Dual energy CT for thoracic applications. Rev Mal Respir. 2012;29(10):1268-1271.

[35] Sadigh G, Kelly AM, Cronin P. Challenges, controversies, and hot topics in pulmonary embolism imaging. AJR Am J Roentgenol. 2011;196(3):497-515. 